\title{
LESSONS LEARNED IN PILOT TESTING SPECIALTY CONSULTATIONS TO BENEFIT INDIVIDUALS WITH LOWER LIMB LOSS
}

CHRISTINE ELNITSKY, PHD, RN, CHNS 1,2,3

GAIL LATLIEF, DO 1,4,5

DEBORAH GAVIN-DRESCHNACK, PHD 1,3

MELANIE HARRIS, CP 4

ROBERT CAMPBELL, PHD, JD 1,3

${ }^{1}$ HeAlTh SERVICES RESEARCH AND DEVELOPMENT \& REHABILITATION RESEARCH AND DEVELOPMENT CENTER OF EXCELLENCE IN MAXIMIZING REHABILITATION OUTCOMES, JAMES A. HALEY VETERANS'

HOSPITAL, TAMPA, FLORIDA

2 Uniformed SerVices University of the Health SCiences, Graduate Program in Nursing, BETHESDA, MARYLAND

${ }^{3}$ Veterans Integrated Service Network 8, Patient Safety Center of inQuiry, James A. Haley

VETERANS' HOSPITAL, TAMPA, FLORIDA

${ }^{4}$ Regional amputation Center, James A. Haley Veterans' Hospital, Tampa, Florida

${ }^{5}$ UNIVERSITY OF SOUTH FLORIDA, COLLEGE OF MEDICINE, TAMPA, FLORIDA

${ }^{6}$ Central Texas Veteran's Affairs Health Care System, Temple, TeXas

7 TeXas A\&M Health SCience Center, COllege of Medicine, DePARTMent of Psychiatry \& BehaVIORAL SCIENCE

\section{ABSTRACT}

Telerehabilitation technologies enable the delivery of rehabilitation services from providers to people with disabilities as well as specialty care consultations. This article discusses the barriers experienced when planning and pilot testing a telerehabilitation multi-site specialty consultation for specialists in their medical centers, and the lessons learned. The barriers included integration and participation, coordination across organizational units, and privacy and information security. Lessons learned included the need for collaboration across multiple departments, telerehabilitation equipment back-ups, and anonymous and private communication protocols. Despite delays resulting from coordination at multiple levels of a national organization, we developed a program plan and successfully implemented a pilot test of the southeast region program. Specialty consultation using telerehabilitation delivery methods requires identifying provider preferences for technological features. Lessons learned could inform development of outpatient telerehabilitation for patients with amputations and studies of patients and providers involved in telerehabilitation.

Keywords: Telerehabilitation, amputation, rehabilitation

\section{INTRODUCTION}

Telerehabilitation, a subset of telehealth, is defined as the delivery of medical rehabilitation services via communication and information technologies (i.e., telephone, email, integrated video and audio teleconferencing) to distant sites (American Telemedicine Association, 2010). Telerehabilitation increases access to quality rehabilitation services by mitigating barriers of distance, time, cost and convenience (Institute of Medicine, 2001; McCue, Fairman, \& Pramuka, 2010) and facilitates patients' independent living in their own homes. It is an "ongoing intervention where users influence its development" (Ekeland, Bowes, \& Flottorp, 2010, p. 11), hence adoption and outcomes are complex collaborative endeavors. Like traditional rehabilitation, telerehabilitation depends on observation, communication, and interaction between the specialists and the patient (Hoenig et al., 2006).

Telerehabilitation provides flexibility in the venue for service delivery at a distance where veterans live, work and participate in the community. Provision of rehabilitation services in the natural home environment is likely to be most effective (McCue et al., 2010; Legg 
\& Langhorne, 2004) and preferred by persons with disabilities (Vladek, Miller, \& Clausen, 1993). Moreover, rehabilitation that occurs in the patient's own home has greater relevance to the patient and promotes the patient's functioning within the context of his/her environment (World Health Organization, 2001; Kulpers, Foster, Smith, \& Fleming, 2009) and improves his/her ability to function independently in the community (Legg \& Langhorn, 2004).

Telerehabilitation can optimize the treatment of patients who have experienced a lower-limb amputation due to vascular or traumatic events. Amputation rehabilitation services comprise a scope of care from assessment to intervention and follow-up services. A range of amputation rehabilitation services, including assessment, monitoring, education, prevention, intervention and followup may be provided. Amputation rehabilitation services typically involve an interdisciplinary team of professionals (e.g., physiatrists, prosthetists, rehabilitation coordinators, therapists, nurses, and assistive technologists).

While telerehabilitation has the potential to improve access and enhance rehabilitation outcomes, we found no studies that have implemented telerehabilitation amputation specialty consultation on a national level. A few studies have described telerehabilitation for individuals with amputations, focusing on technical acceptability of home-based videos for wound assessment during consultations (Rintala et al., 2004) or home-based program to support independent living in the community (Bendixen et al., 2008), or a meta-analysis of telephone-based counseling for patients with a variety of physical disabilities including limb amputation (Dorstyn, Mathias, \& Denson, 2011). In a feasibility study (Hoenig et al., 2006), both patients and providers expressed satisfaction with the telerehabilitation delivery system given its ease of use and ability to assess patients in the context of their home environments. Despite growing investment in innovative technology, little is known regarding the effects and costs of telerehabilitation (Currell, Urquhart, Wainwright, \& Lewis, 2000), nor the experiences of providers with telerehabilitation technology.

A few studies have identified barriers to and lessons learned from implementing telerehabilitation (Barrett, Larson, Carville, \& Ellis, 2009; Chumbler, Rose et al., 2010; Chumbler, Quigley et al., 2010). Barriers have included challenges related to changes in technology (Chumbler, Quigley et al., 2010b), selection, and insufficient training (Chumbler, Rose et al., 2010; Barrett et al., 2009); equipment functionality (Chumbler, Quigley et al., 2010); reliability and human resource support (Barrett et al., 2009; Chumbler, Rose et al., 2010); coordination (Barrett et al., 2009, Chumbler, Rose et al., 2010) and interdepartmental integration (Chumbler, Quigley et al., 2010) and protection of human subjects (Chumbler, Rose et al., 2010; Chumbler, Quigley et al., 2010). The purpose of this paper is to present barriers and lessons learned based on the development and pilot testing of an amputation rehabilitation specialty consultation program. While the operations quality improvement project addressed amputation rehabilitation, findings could inform development and implementation of other telerehabilitation programs and patient populations. We will briefly describe the context of the project, telerehabilitation lessons learned (i.e., technology challenges), and lessons learned specific to a national program (i.e., cross-regional coordination and information security challenges).

\section{DESCRIPTION OF TELEREHABILITATION \\ SPECIALTY CARE CONSULTATION FOR PATIENTS WITH LIMB AMPUTATIONS}

The Department of Veterans Affairs (VA) provides the infrastructure with its telemedicine programs and equipment at $153 \mathrm{VA}$ medical centers and hundreds of community based clinics (CBOC) and the ability to link with the videoconferencing network to enable telerehabilitation to be provided to veterans within communities nationwide. In 2009, the VA Amputation System of Care began providing amputation rehabilitation clinic visits via telerehabilitation technologies. In 2011, VA specialty care began transitioning to outpatient settings (Department of Veterans Affairs, 2011a, 2011b) with pilot tests of synchronous consultations between specialists and primary care providers in rural areas. VA began implementing telerehabilitation specialty care access network (SCAN) consultations for veterans who might previously have been referred for office visits requiring veterans to drive sometimes long distances to the appointments (Department of Veterans Affairs, 2011a, 2011b). The purpose of this pilot project was to develop a plan for implementation of specialty care consultations using telerehabilitation technologies to veterans' primary care providers in CBOCs across the southeastern United States and pilot test the implementation methods.

The VA SCAN program is based on the Project ECHO (Extension for Community Healthcare Options) program model at the University of New Mexico, which began in 2004. Project ECHO linked urban healthcare specialists with primary care providers in rural communities, providing access to specialist expertise the providers needed to better treat their patients (Arora et al., 2011). Rather than teleconferencing a specialist with a patient, $\mathrm{ECHO}$ used telemedicine technologies and clinical management tools to link specialists with groups of primary care providers to obtain specialty consultation, mentorship and continuing medical education. $\mathrm{ECHO}$ developed the primary care providers' knowledge and efficacy in treating various diseases. These primary care providers could then co-manage care for patients with complex health conditions with specialists (Arora et al., 2011). 
Project ECHO program model elements for the implementation of specialty teleconsultations (Arora et al., 2007) are outlined in Table 1.

\section{TABLE 1: PROGRAM ELEMENTS FOR} IMPLEMENTING SPECIALTY TELECONSULTATIONS transfemoral amputation. The subject's primary care physician and physiatrist approved for him to be evaluated for a prosthetic knee and additional components as needed to ambulate and perform running activities.

Additionally, the patient was interested in recreational jogging and playing basketball on a sloped driveway. His amputation was of traumatic origin. Functionally, he could descend stairs with reciprocal foot pattern. He was able to walk on a treadmill. His 6 minute walk was timed at approximately 2.7 mph.

\begin{tabular}{|l|l|}
\hline $\begin{array}{l}\text { Principle } \\
\begin{array}{l}\text { 1. Disease is appropriate to treat using a knowledge } \\
\text { network approach }\end{array}\end{array}$ & $\begin{array}{l}\text { Description } \\
\text { Select a disease that is common, has complex } \\
\text { management, has high social impact, treatment is } \\
\text { evolving, serious outcomes if fail to treat the disease, } \\
\text { improved outcomes will occur with disease } \\
\text { management }\end{array}$ \\
$\begin{array}{ll}\text { 2. Telemedicine as a learning technology } \\
\text { - Use telemedicine to optimize limited specialty health } \\
\text { care resources. Use electronic information and } \\
\text { communication technologies to provide and support } \\
\text { health care. }\end{array}$ \\
$\begin{array}{l}\text { Case-based learning with co-management of } \\
\text { patients by primary care provider and specialists. }\end{array}$ \\
4. Centralized database & $\begin{array}{l}\text { Coordinate care through a Health } \\
\text { Insurance Portability and Accountability } \\
\text { Act (HIPAA)-compliant database }\end{array}$ \\
\hline
\end{tabular}

\section{DESCRIPTION OF TECHNOLOGY}

The system used to support telerehabilitation specialty consultation services in the VA was built around a national network of integrated video and audio units with color monitors with pan/tilt/zoom cameras and video transmission media using Integrated Services Digital Network (ISDN) installed in Veterans Affairs Medical Centers (VAMCs) of service providers. A telerehabilitation (TR) consultation session was conducted with 26 participating specialists at 7 sites nationwide.

\section{TElEREHABILITATION CASE DESCRIPTION}

As the telerehabilitation program is focused on providing specialty clinical consultation by specialists to primary care providers in CBOCs, information about specific cases must be provided to the specialist by a primary care provider for the consultation. An intake form was developed for the purpose of communicating anonymous clinical information per the clinical practice guidelines for telemedicine/telerehabilitation. The case patient was a 35 year old male veteran of Operations Iraqi Freedom/ Enduring Freedom with a unilateral left

\section{IMPLEMENTATION BARRIERS}

The project team experienced barriers related to the program planning and the implementation of the pilot test. These barriers presented delays while informing the development of the program and test and requiring unanticipated changes in the original plans. The barriers included provider integration and participation, coordination across departments, and privacy and information security. 


\section{TABLE 2: BARRIERS IN IMPLEMENTATION OF TR SPECIALTY CONSUlTATION PILOT TEST}

\begin{tabular}{|c|c|}
\hline Barrier & Description \\
\hline 1. Integration and Participation & $\begin{array}{l}\text { - Provider days and times of availability vary } \\
\text { - Provider interest in specific specialty care topics vary }\end{array}$ \\
\hline 2. Coordination Across Organizational Units & $\begin{array}{l}2 \text { months to clarify IT equipment and bridge schedule } \\
6 \text { months across } 3 \text { different organizational levels to } \\
\text { coordinate clinical funding } \\
\text { 3-6 months across different organizational levels to } \\
\text { coordinate policies on clinical privileges, workload } \\
\text { credit, continuing education credit }\end{array}$ \\
\hline 3. Privacy and Information Security & $\begin{array}{l}\text { - Telerehabilitation patient case study information } \\
\text { - Information communication protocol }\end{array}$ \\
\hline
\end{tabular}

\section{INTEGRATION AND PARTICIPATION}

Introducing telerehabilitation specialty consultations potentially changes the current workflow of providers in the CBOCs and specialists in the medical centers. Primary care providers are scheduled to see patients in the CBOCs throughout the day and may not be available to participate, particularly if there is no workload credit. The southeast region is, in fact, the busiest with the greatest demand for appointments. Hence there was a high likelihood of limited participation. Barriers included provider availability and time required to prepare for the specific consultation topic(s). Depending upon the provider's patient population, individual providers may have more or less interest in specific specialty topics.

\section{COORDINATION ACROSS ORGANIZATIONAL UNITS}

It was critical to coordinate across units and departments within the organization and levels of the organization (e.g., national, regional, and local facility). Coordination across clinical operations, project team and information technology departments and organizational levels required time. Likewise, development of policies and logistics across primary care and specialty care services was required at various levels of the organization. Obtaining continuing medical education credit for providers in the pilot and subsequent sessions required coordination across levels of the organization.

\section{PRIVACY AND INFORMATION SECURITY}

The primary care provider must share specific patient case information with the specialist who conducts the assessment prior to the scheduled telerehabilitation consultation. Both professionals are responsible for maintaining the confidentiality and privacy of the patient's records. While VA has secure electronic health records, these records are not easily accessible to providers at VA facilities outside the patient's designated VA site.

\section{LESSONS LEARNED}

The project team developed a variety of solutions to address the barriers we encountered. Table 2 depicts each barrier, followed by the solutions identified for the barrier. 


\section{TABLE 3: SOLUTIONS TO BARRIERS IN}

\section{TELEREHABILITATION IMPLEMENTATION}

Barrier

1. Integration and Participation

2. Coordination across Organizational Units

3. Privacy and Information Security
Planning Solutions

Schedule regular recurring telerehabilitation consultations on primary care providers' preferred topics

Collaborate with providers to identify the most convenient day/time for the majority of providers

Arrange SCAN networks as regional in scope due to time zones

Provide calendar of events on a common access web site or SharePoint site

Identify strong champions at each site

Train project staff and develop project materials Develop project plans for future project roll-out Allocate pilot project personnel

Execute clinical privilege agreements

Establish Information technology back-up strategies Arrange for workload credit

Provide continuing medical education

Establish communication protocol

Draft anonymous case information form

\section{INTEGRATION AND PARTICIPATION}

It was important to identify and begin collaborating with those affected by the specialty consultations early in the project. Project team members communicated with and invited participation of primary care providers and specialists in the planning phase. The pilot test and subsequent sessions were planned based on an assessment of their needs. Participation necessarily involved the clinic manager who scheduled patient appointments; therefore, collaboration with the managers was a high priority. Having determined the best time of day for providers to attend consultations was noon or late afternoon, scheduling at these times would facilitate participation by the greatest number of attendees. Our protocol for the pilot test allowed scheduling at noon. We communicated with providers to identify their preferred topic(s) and an anonymous case description for the telerehabilitation specialty consultation.

Twenty-six providers from seven different states attended the pilot test, the largest attendance of any single SCAN teleconsultation in the first year. For the larger program, we planned to provide a monthly calendar of consultation topics and their scheduled times posted on our SCAN network site. Monthly calendar email reminders would be sent to potential participants. We identified strong champions at $\mathrm{CBOC}$ sites to communicate preferences to the project team and facilitate and encourage participation.

\section{COORDINATION ACROSS ORGANIZATIONAL UNITS}

Implementing programs requires coordination of multiple departments (e.g., clinical operations at the national and local levels, information technology, fiscal, legal). Implementation required training and development of project materials, development of project plans for future project roll-out, and allocation of pilot project personnel. Simultaneously, policy development (e.g., clinical privileges for the specialist at a different site) was required at multiple levels before the pilot test. Coordination with information technology (IT) and the communications department was required to access the bridge connections and a simultaneous toll free conference call line as back-up for 26 different participants in seven different states. Coordination with national policy makers and legal was required to obtain workload credit for providers given the statutory requirements for veterans care services. Coordination with local and regional levels was required to obtain continuing medical education credits for clinician participants in the pilot test. 


\section{PRIVACY AND INFORMATION SECURITY}

Telerehabilitation equipment (i.e., conference call and integrated video conferencing equipment) was considered in the development of procedures for the provider to share specific information relevant to the case with the specialist(s) while minimizing the risk of disclosure of telerehabilitation patient case study information. Both an information communication protocol and information form about the case were developed by the project team. Secure encrypted email was used by the provider to communicate anonymous information about the case to the specialist prior to the consultation. Neither the patient nor any personally identifiable information were part of the pilot test.

\section{DISCUSSION AND RECOMMENDATIONS}

This article presents three barriers to the development and pilot testing of a telerehabilitation specialty consultation program. The barriers include integration and participation, coordination across multiple departments, and privacy and information security. Despite delays associated with planning a new program and coordination across departments, we effectively developed a plan and successfully implemented a pilot test of the southeast region SCAN program. (Table 3)

Based on our experience, we recommend that organizations planning and implementing telerehabilitation programs use a similar approach to development wherein the providers' perspectives on needs, schedule, and topics are first ascertained. With this information, program planners can apply a collaborative and iterative approach to development throughout the process. Coordination across multiple departments and multiple levels of complex health care organizations requires advanced planning and pilot testing ranging from single site tests to multi-site, multi-phase testing to identify barriers and develop adequate solutions with consultation across various groups and sites.

The use of telerehabilitation cost effectively addresses patient-centered needs and healthcare staff shortages. Telerehabilitation for patients with limb amputations is an innovative way to meet the rehabilitation and community reintegration needs of patients and their families in timely and convenient ways. Our findings suggest that the development, implementation and future evaluation of telerehabilitation specialty consultations are highly complex. As reported in previous studies, such complexity is often underappreciated and may not be reported (May et al., 2002).

\section{ACKNOWLEDGEMENTS}

The views expressed in this manuscript are those of the authors and do not reflect the official policy or position of the Department of Veterans Affairs or the United States Government. Partial funding was provided by the Department of Veterans Affairs (IMA00460718). 


\section{REFERENCES}

1. American Telemedicine Association. (2010). A blueprint for telerehabilitation guidelines. Retrieved from http:// www.americantelemed.org/files/public/standards/ ATA\%20Telerehab\%20Guidelines\%20v1\%20(2).pdf

2. Arora, S., Geppert, C.M., Kalishman. S., Dion. D., Pullara, F., Bjeletich, B.,...Scaletti, J.V. (2007). Academic health center management of chronic diseases through knowledge networks: Project ECHO. Academic Medicine, 82, 154-160.

3. Arora, S., Kalishman, S., Dion, D., Som, D., Thornton, K., Bankhurst, A.,...Yutzy S. (2011). Partnering urban academic medical centers and rural primary care clinicians to provide complex chronic disease care. Health Affairs, 30, 1176- 1184.

4. Barrett, M., Larson, A., Carville, K., \& Ellis, I. (2009). Challenges faced in implementation of a telehealth enabled chronic wound care system. Rural Remote Health, 9(3), 1154. Retrieved from http://www.rrh.org.au/ articles/subviewnew.asp?ArticlelD=1154

5. Bendixen, R.M., Levy, C., Lutz, B.J., Horn, K.R., Chronister, K., \& Mann, W.C. (2008). A telerehabilitation model for victims of polytrauma. Rehabilitation Nursing Journal, 33, 215-220.

6. Chumbler, N.R., Rose, D.K., Griffiths, P., Quigley, P., McGee-Hernandez, N., Carlson, K.A., ...Hoenig H. (2010). Study protocol: Home-based telehealth stroke care: A randomized trial for veterans. Trials,11, 74 . doi: 10.1186/1745-6215-11-74

7. Chumbler, N.R., Quigley, P., Sanford, J., Griffiths, P., Rose, D., Morey, M., ...Hoenig, H. (2010). Implementing telerehabilitation research for stroke rehabilitation with community dwelling veterans: Lessons learned. International Journal of Telerehabilitation, 2(1), 15-21.

8. Currell, R., Urquhart, C., Wainwright, P., \& Lewis, R. (2000, January 20). Telemedicine versus face to face patient care: Effects on professional practice and health care outcomes. Cochrane Database Systematic Review, (2): doi: 10.1002/14651858.CD002098

9. Department of Veterans Affairs. (2011a). DVA Volume II Medical programs and Information Technology Programs, Congressional Submission, FY2012 Funding and FY2013 Advance Appropriations Request. Washington D.C.: DVA.

10. Department of Veterans Affairs. (2011b). Strategic plan refresh fiscal year (FY) 2011-2015. Washington, D.C.: DVA. Retrieved from http://www.va.gov/VA_2011-2015_ Strategic_Plan_Refresh_wv.pdf

11. Dorstyn, D.S., Mathias, J.L., \& Denson, L.A. (2011). Psychosocial outcomes of telephone- based counseling for adults with an acquired physical disability: A metaanalysis. Rehabilitation Psychology, 56(1), 1-14. doi: 10.1037/a0022249

12. Ekeland, A.E., Bowes, A., \& Flottorp, S. (2010). Effectiveness of telehealth: A systematic review of reviews. International Journal of Medical Informatics, 79, 736-771.

13. Hoenig, H., Sanford, J.A., Butterfield, T., Griffiths, P.C., Richardson, P., \& Hargaves, K. (2006). Development of teletechnology protocol for in-home rehabilitation. Journal of Rehabilitation Research \& Development, 43, 287-298.
14. Institute of Medicine . (2001). Crossing the quality chasm. Washington, DC: National Academy Press.

15. Kulpers, P., Foster, M., Smith, S., \& Fleming, J. (2009). Using ICF-environment factors to enhance the continuum of outpatient $A B I$ rehabilitation: $A n$ exploratory study. Disability and Rehabilitation, 31, 144151. doi:10.1080/01674820701817938.

16. Legg, L., \& Langhorn, P. (2004). Rehabilitation therapy services for stroke patients living at home: A systematic review of randomised trials. Lancet, 363, 352-356.

17. May, C.R., Williams, T.L., Mair, F.S., Mort, M.M., Shaw, N.T., \& Gask, L. (2002). Factors influencing the evaluation of telehealth interventions: preliminary results from a qualitative study of evaluation projects in the UK. Journal of Telemedicine and Telecare, 8(Suppl 2), 65-67. doi:10.1258/jtt.2012.120219.

18. McCue, M., Fairman, A., \& Pramuka, M. (2010). Enhancing quality of life through telerehabilitation. Physical Medicine Rehabilitation Clinics of North America, 21, 195-205.

19. Rintala, D.H., Krouskop, T.A., Wright, J.V., Garber, S.L., Frnka, J., Henson, H.K.,... Monga, T.N. (2004). Telerehabilitation for veterans with a lower-limb amputation or ulcer: Technical acceptability of data. Journal of Rehabilitation Research and Development, 41, 481-490.

20. Vladek, B.C., Miller, N.A., \& Clausen, S.B. (1993). The changing face of long-term care. Health Care Finance Review, 14(4), 5-23.

21. World Health Organization. (2001). International classification of functioning, disability and health (ICF). Geneva: WHO. 
